\title{
Der Beitrag von Keratinozyten an der Pathogenese des kutanen Lupus erythematodes
}

\section{Contribution of Keratinocytes to the Pathogenesis of Cutaneous Lupus Erythematosus}

Autor

Institut

\section{Wittmann}

Klinik und Poliklinik für Dermatologie und Venerologie der Medizinischen Hochschule Hannover

\section{Bibliografie}

Dol $10.1055 / \mathrm{s}-2007-967005$

Akt Dermatol 2007; 33:

413-416 @ Georg Thieme

Verlag KG Stuttgart · New York ISSN 0340-2541

Korrespondenzadresse

Prof. Dr. med. Miriam Wittmann

Klinik und Poliklinik für Dermatologie und Venerologie der Medizinischen Hochschule Hannover

Ricklinger Straße 5 30449 Hannover Wittmann.Miriam@ MH-Hannover.de

\section{Zusammenfassung \\ $\nabla$}

Hautveränderungen sind eine häufige Manifestationsform der Autoimmunerkrankung Lupus erythematodes (LE). Derzeit steht keine kurative Therapie zur Verfügung. Eine Voraussetzung für die Entwicklung einer effektiven Lokaltherapie besteht in einem besseren Verständnis der zugrunde liegenden Pathomechanismen. Keratinozyten werden als Zielzellen des auto-aggressiven

Ein Großteil der Patienten, die an einem chronisch diskoiden oder subakut kutanen LE leiden, erreicht mit konsequentem Lichtschutz, Lokaltherapie und gegebenenfalls Hydroxychloroquin einen stabilen Hautzustand oder Erscheinungsfreiheit. Dennoch bleiben eine Reihe von Patienten, bei denen eine weitergehende systemische Therapie notwendig wird. Es besteht Bedarf für eine verbesserte und effektive Lokaltherapie sowie für Marker, die auf Wirkstoff oder die Wirkstoffkombination hinweisen, die für den jeweiligen Patienten den besten Therapieerfolg erbringen.

Die Pathophysiologie des Lupus erythematodes (LE) ist nach wie vor nur unvollständig verstanden. Untersuchungen werden auch durch die unterschiedlichen klinischen Ausprägungen und Verlaufsformen erschwert. Es kommt auf der Grundlage eines empfänglichen genetischen Hintergrundes zur immunologischen Fehlregulation. Eine große Anzahl von Suszeptibilitätsgenen [1] wurde beschrieben, deren Bedeutung jedoch nicht immer in unterschiedlichen Kohorten bestätigt werden konnte. Derzeit wird ein Beitrag von Varianten des IRF5-Gens für die Lupus-Pathogenese diskutiert [2,3].

\footnotetext{
* Förderung durch die Deutsche Forschungsgemeinschaft (Wi1822/5-1).

Vortrag beim wissenschaftlichen Jahres-Symposium der Berliner Stiftung für Dermatologie, 12. Mai 2007, Berlin.
}

Angriffs gesehen. Es mehren sich jedoch die Hinweise, dass Keratinozyten auch - mittels Mediatorfreisetzung - aktiv an der Regulation und Aufrechterhaltung der Lupuspathologie beteiligt sind. In der vorgestellten Untersuchung haben wir das pro-entzündliche Antwortmuster von Keratinozyten von LE-Patienten im Vergleich zu Keratinozyten von gesunden Spendern untersucht.

In dem Bemühen, therapeutische Zielstrukturen bzw. Aktivitätsmarker zu identifizieren, wurde die Expression von proinflammatorischen bzw. immunregulatorischen Zytokinen untersucht. Es liegt eine Vielzahl von Untersuchungen vor, die bei Patienten mit LE Zytokine, insbesondere im Serum, bestimmt haben und darin Hinweise für Krankheitsaktivität oder auch Therapieansprechen sehen. Ein wesentlicher Anteil der publizierten Daten bezieht sich auf den systemischen LE. Es sind u.a. folgende Zytokine mit einer erhöhten Krankheitsaktivität in Zusammenhang gebracht worden: IL-6 [4-6], IL-10 [7], sowie auch in läsionaler Haut erhöht gefunden: Typ I-Interferone [8,9], IL-18 [10-12], [13,14], TNF $\alpha$ [15-17] und high mobility group box chromosomal protein 1 (HMGB-1) [16]. Weiterhin mehren sich die Hinweise, dass insbesondere bei Patienten mit systemischem LE Interferon-responsive-Gene hochreguliert werden, was als „Interferon-Signature“ in mRNA-basierten Mikroarrays nachgewiesen, aber auch in läsionaler Haut [18] gezeigt wurde. Es besteht eine Korrelation zwischen dieser „Interferon-Signature“ und der Krankheitsaktivität [19].

Seit Langem wird die so genannte „Clearance Hypothese“ für den LE diskutiert, welche besagt, dass vermehrt anfallende apoptotische Zellen nicht im „geordneten“ Maß und, wie physiologischerweise üblich, entzündungsfrei eleminiert 
werden. Dies kann entweder damit zusammenhängen, dass zu viel apototisches Material vorliegt oder dass die abbauenden Mechanismen gestört sind. Für beides gibt es Hinweise. Beim LE fallen - soweit vorhanden - insbesondere Antikörper auf, die gegen nukleäre, also intrazelluläre Antigene gerichtet sind. Bei einem „geordnet“ ablaufenden apoptotischen Zelltod werden solche Antigene dem Immunsystem nicht zugänglich. Im Gegensatz dazu geht man bei LE davon aus, dass die ehemals intrazellulär gelegenen Antigene an B- und T-Zellen präsentiert werden und somit den Autoimmunprozess triggern können.

In den letzten Jahren ist von unterschiedlichen Diziplinen her intensiv auf dem Gebiet der regulatorischen T-Zellen geforscht worden. Hierdurch ist ein verändertes Verständnis für die periphere Toleranz als ergänzender Mechanismus zur zentralen Toleranz entstanden. Es scheint, dass eine lebenslange, aktive periphere Toleranz notwendig ist [20], um autoreaktive Zellen, die jeder in sich trägt, in Schach zu halten. Für den LE sind einige Arbeiten erschienen, die auf eine Verminderung bzw. verminderte Aktivität der regulatorischen T-Zellen hinweisen [21,22]. In der Durchbrechung der Toleranz (vermittelt u.a. über unreife DC oder plasmazytoide DC) kommt wohl den erhöht gefundenen Typ I-Interferonen ebenfalls eine wichtige Rolle zu [23].

\section{Die Hautentziindung}

$\nabla$

Die Haut ist bei allen bekannten Formen des LE ein wichtiges Zielorgan. Obwohl die Hautveränderungen morphologisch unterschiedlich erscheinen, vergleicht man den chronisch diskoiden, subakut kutanen und systemischen LE, so sind dennoch immunhistologisch ähnliche Veränderungen zu beobachten. Eine Interfacedermatitis mit vakuoliger Degeneration im Bereich der Basalschicht der Keratinozyten und eine vorwiegend lymphozytäre Infiltration in den Bereichen des dermo-epidermalen Übergangs sind häufig zu finden.

Drei wesentliche pathophysiologisch vermutlich relevante Veränderungen bezüglich der Keratinozyten sind seit Längerem bekannt:

1. Es findet sich eine Neigung zum apoptotischen Zelltod [24-27]. (Es ist anzumerken, dass eine kürzlich publizierte Arbeit keine vermehrte Apoptoserate in UV-exponierter Haut von LE-Patienten finden konnte [28]).

2. Es finden sich Anzeichen einer Zellaktivierung, insbesondere in Form einer Hochregulation immunologisch wichtiger

\begin{tabular}{|c|c|}
\hline & „gesunde“ Keratinozyten \\
\hline $\begin{array}{l}\text { Sterbeverhalten } \\
\text { (Apoptose) }\end{array}$ & $\begin{array}{l}\text { zeigen Apoptose nach Einfluss von } \\
\text { UV-Licht und bestimmten Entzün- } \\
\text { dungsbotenstoffen wie TNF } \alpha\end{array}$ \\
\hline Schutz vor Apoptose & $\begin{array}{l}\text { gegeben u.a. durch Mitglieder der } \\
\text { Interleukin-12-Familie }\end{array}$ \\
\hline $\begin{array}{l}\text { Produktion von Zytokinen } \\
\text { im Ruhezustand }\end{array}$ & $\begin{array}{l}\text { im Ruhezustand werden einige } \\
\text { Chemokine, entzündungslimitierende } \\
\text { Stoffe und nur sehr geringe Mengen an } \\
\text { pro-inflammatorischen Mediatoren } \\
\text { sezerniert }\end{array}$ \\
\hline $\begin{array}{l}\text { Produktion von Zytokinen } \\
\text { nach Aktivierung }\end{array}$ & $\begin{array}{l}\text { pro-inflammatorische Mediatoren } \\
\text { werden gleichzeitig mit vermehrten } \\
\text { „Schutz-“ und anti-inflammatorischen } \\
\text { Botenstoffen produziert }\end{array}$ \\
\hline
\end{tabular}

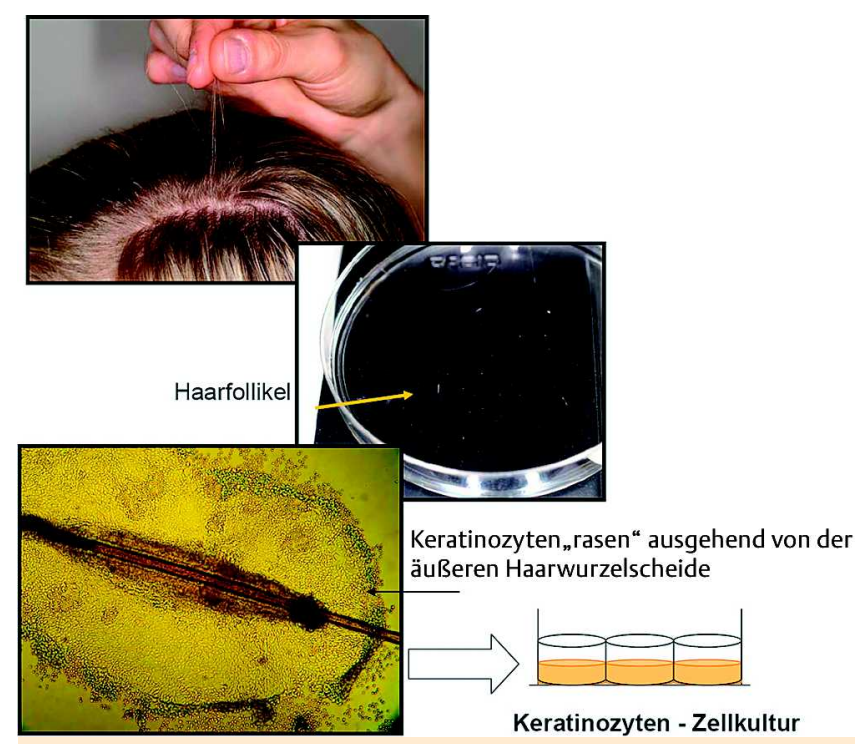

Abb. 1 Patientenbezogene Keratinozyten werden gewonnen, indem sie aus epidermalen Stammzellen der äußeren Haarwurzelscheide angezüchtet werden.

Oberflächenmoleküle [29-32] und der Produktion von Chemokinen [33] und proinflammatorischen Zytokinen (s. o.).

3. Es kommt zur Translokation von nukleären Antigenen an die Zelloberfläche. Dies ist insbesondere für Ro (SSA) gezeigt. Diese Translokation ist durch UVB-Licht [24,34] und TNF $\alpha$ $[35,36]$ auslösbar. Es wird vermutet, dass die so an die Zelloberfläche gelangten Autoantigene durch das Immunsystem erkannt werden können.

Eine wichtige Rolle für das Zustandekommen und der Chronifizierung der kutanen LE-Symptome ist vermutlich in der „entzündlichen“ Lokalantwort zu sehen. Aktivierte Keratinozyten sind potente Produzenten von Chemokinen und Zytokinen [37]. Diese wiederum wirken auf Nachbarzellen (z.B. professionelle APC), aber auch chemotaktisch dahin $[33,38]$, vermehrt Blutleukozyten in die oberen Hautschichten zu dirigieren.

Mit unserer Arbeit wollten wir die Frage beantworten, ob Keratinozyten von LE-Patienten im Vergleich zu Normalkeratinozyten ein anderes proinflammatorisches Verhalten zeigen. Hierfür haben wir Keratinozyten aus epidermalen Stammzellen der äußeren Haarwurzelscheide [39] isoliert ( $\bullet$ Abb. 1). Wir haben also Patientenzellen untersucht, die aus nicht-läsionaler Haut stam-
„Lupus“-Keratinozyten

zeigen eine basal etwas erhöhte Apoptoserate;

sind empfindlicher gegenüber Entzündungsbotenstoffen insbesondere gegenüber IL-18 Schutz erfolgt im gleichen prozentualen Ausmaß wie bei Gesunden erhöhte Produktion von pro-inflammatorischen Mediatoren auch durch nicht stimulierte/aktivierte Zellen

vermehrte Produktion pro-inflammatorischer Botenstoffen, insbesondere auch TNF $\alpha$ und wenig „Schutzzytokine“, z. B. der IL-12-Familie
Tab. 1 Unterschiede zwischen Keratinozyten von Lupus erythematodes (LE)-Patienten und gesunden Kontrollpersonen 


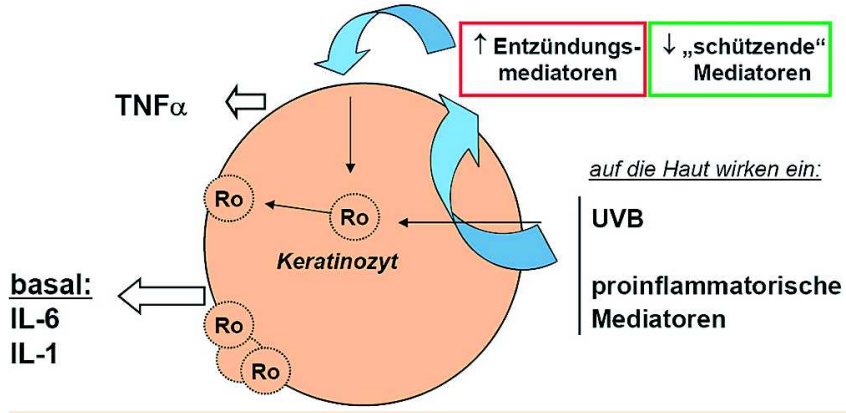

Abb. 2 Beitrag von Keratinozyten an der Pathogenese des LE. Von außen einwirkendes UV-Licht oder pro-entzündliche Botenstoffe führen dazu, dass Hautzellen von LE-Patienten ebenfalls vermehrt proinflammatorische Botenstoffe freisetzen, die wiederum auf andere Keratinozyten wirken. Gleichzeitig bleibt die Produktion von schützenden Mediatoren aus. Unter diesem Einfluss sterben die Zellen vermehrt. Es kommt neben der Produktion von entzündungsfördernden Botenstoffen auch zum Transport von „Autoantigenen“ (hier beispielhaft Ro dargestellt) an die Zelloberfläche, wo sie durch das Immunsystem erkannt werden können.

men. Die bisherigen Ergebnisse dieser Untersuchungen sind in - Tab. 1 aufgeführt. Wir konnten an isolierten Hautzellen zeigen, dass Keratinozyten von LE-Patienten in einer „Entzündungsumgebung“ vermehrt in den apoptotischen Zelltod gehen. Weiterhin produzieren diese Zellen auch basal größere Mengen von pro-inflammatorischen Mediatoren und scheinen sich in einem selbstverstärkenden Rückkopplungskreislauf gegenseitig zu aktivieren. Problematisch in dieser Situation erscheint die verminderte Produktion von Botenstoffen, die einen vermehrten Zelltod verhindern [40] wie u.a. IL-12 ( Abb. 2). Unsere bisherigen Ergebnisse unterstützen die Annahme, dass Keratinozyten eine durchaus aktive Rolle bei der Krankheitsentstehung und -aufrechterhaltung des LE spielen.

Wie können diese Erkenntnisse das therapeutische Vorgehen beeinflussen und optimieren? Zum einen erscheint die bislang häufig eingesetzte Therapie mit (Hydroxy)Chloroquin auch positiv im Sinne der hier vorgestellten Erkenntisse zur epidermalen Entzündung zu wirken. So konnten Wozniacka u. Mitarb. eine verminderte TNF $\alpha$, IL-6 und IL-18 Expression (im Serum) unter Hydroxychloroquin Therapie finden [41]. Dennoch fehlt eine effektive, topisch anwendbare immunmodulatorische Therapie. Für die genaue Identifizierung therapeutischer Zielstrukturen in der entzündeten Haut ist wichtig, die Hierarchie der proinflammatorischen Botenstoffen noch besser zu identifizieren. Daher besteht klarer Forschungsbedarf, die epidermale Pathogenese des LE weiter aufzuklären.

\section{Abstract}

\section{Contribution of Keratinocytes to the Pathogenesis of Cutaneous Lupus Erythematosus}

$\nabla$

Lupus erythematosus (LE) is an autoimmune disorder and cutaneous manifestations belong to the most common clinical features of LE. Up to now, there exists no causative treatment regimen. However, a prerequisite for development of effective local therapeutics is the better understanding of the underlying pathophysiological mechanisms. In cutaneous LE (CLE), keratinocytes are considered to be target cells for immunological injury. Next to their role as target cells, evidence is accumulating that keratinocytes play an important role in actively regulating and maintaining the pathology in CLE by virtue of mediator release. In this study, we were interested in determining differences in the proinflammatory response of patient-derived keratinocytes.

\section{Literatur}

1 Alarcon-Riquelme ME. The genetics of systemic lupus erythematosus: understanding how SNPs confer disease susceptibility. Springer Semin Immunopathol 2006; 28: 109-117

2 Graham RR, Kozyrev SV, Baechler EC, Reddy MV, Plenge RM, Bauer JW, Ortmann WA, Koeuth T, Gonzalez Escribano MF, Pons-Estel B, Petri M, Daly M, Gregersen PK, Martin J, Altshuler D, Behrens TW, Alarcon-Riquelme ME. A common haplotype of interferon regulatory factor 5 (IRF5) regulates splicing and expression and is associated with increased risk of systemic lupus erythematosus. Nat Genet 2006; 38: $550-555$

3 Graham RR, Kyogoku C, Sigurdsson S, Vlasova IA, Davies LR, Baechler EC, Plenge RM, Koeuth T, Ortmann WA, Hom G, Bauer JW, Gillett C, Burtt N, Cunninghame Graham DS, Onofrio R, Petri M, Gunnarsson I, Svenungsson E, Ronnblom L, Nordmark G, Gregersen PK, Moser K, Gaffney PM, Criswell LA, Vyse TJ, Syvanen AC, Bohjanen PR, Daly MJ, Behrens TW, Altshuler $D$. Three functional variants of IFN regulatory factor 5 (IRF5) define risk and protective haplotypes for human lupus. Proc Natl Acad Sci USA 2007; 104: 6758-6763

4 Asanuma Y, Chung CP, Oeser A, Shintani A, Stanley E, Raggi P, Stein CM. Increased concentration of proatherogenic inflammatory cytokines in systemic lupus erythematosus: relationship to cardiovascular risk factors. J Rheumatol 2006; 33: 539-545

5 Davas EM, Tsirogianni A, Kappou I, Karamitsos D, Economidou I, Dantis PC. Serum IL-6, TNFalpha, p55 srTNFalpha, p75srTNFalpha, srIL-2alpha levels and disease activity in systemic lupus erythematosus. Clin Rheumatol 1999; 18: 17-22

6 Linker-Israeli M, Deans RJ, Wallace DJ, Prehn J, Ozeri-Chen T, Klinenberg $J R$. Elevated levels of endogenous IL-6 in systemic lupus erythematosus. A putative role in pathogenesis. J Immunol 1991; 147: 117- 123

7 Lauwerys BR, Garot N, Renauld JC, Houssiau FA. Interleukin-10 blockade corrects impaired in vitro cellular immune responses of systemic lupus erythematosus patients. Arthritis Rheum 2000; 43: 1976- 1981

8 Farkas L, Beiske K, Lund-Johansen F, Brandtzaeg P, Jahnsen FL. Plasmacytoid dendritic cells (natural interferon-alpha/beta-producing cells) accumulate in cutaneous lupus erythematosus lesions. Am J Pathol 2001; 159: 237-243

9 Nousari HC, Kimyai-Asadi A, Tausk FA. Subacute cutaneous lupus erythematosus associated with interferon beta-1a. Lancet 1998; 352: $1825-1826$

10 Wong CK, Li EK, Ho CY, Lam CW. Elevation of plasma interleukin-18 concentration is correlated with disease activity in systemic lupus erythematosus. Rheumatology (Oxford) 2000; 39: 1078-1081

11 Wong CK, Ho CY, Li EK, Tam LS, Lam CW. Elevated production of interleukin-18 is associated with renal disease in patients with systemic lupus erythematosus. Clin Exp Immunol 2002; 130: 345 - 351

12 Amerio P, Frezzolini A, Abeni D, Teofoli P, Girardelli CR, De Pita O, Puddu $P$. Increased IL-18 in patients with systemic lupus erythematosus: relations with $\mathrm{Th}-1$, Th-2, pro-inflammatory cytokines and disease activity. IL-18 is a marker of disease activity but does not correlate with pro-inflammatory cytokines. Clin Exp Rheumatol 2002; 20: $535-538$

13 Calvani N, Richards HB, Tucci M, Pannarale G, Silvestris F. Up-regulation of IL-18 and predominance of a Th1 immune response is a hallmark of lupus nephritis. Clin Exp Immunol 2004; 138: 171 - 178

14 Park MC, Park YB, Lee SK. Elevated interleukin-18 levels correlated with disease activity in systemic lupus erythematosus. Clin Rheumatol 2004; 23: 225-229

15 Toro JR, Finlay D, Dou X, Zheng SC, LeBoit PE, Connolly MK. Detection of type 1 cytokines in discoid lupus erythematosus. Arch Dermatol 2000; 136: $1497-1501$

16 Popovic K, Ek M, Espinosa A, Padyukov L, Harris HE, Wahren-Herlenius $M$, Nyberg $F$. Increased expression of the novel proinflammatory cytokine high mobility group box chromosomal protein 1 in skin lesions of patients with lupus erythematosus. Arthritis Rheum 2005; 52: 3639 3645 
17 Zampieri S, Alaibac M, Iaccarino L, Rondinone R, Ghirardello A, SarziPuttini P, Peserico A, Doria A. Tumour necrosis factor alpha is expressed in refractory skin lesions from patients with subacute cutaneous lupus erythematosus. Ann Rheum Dis 2006; 65: 545-548

18 Wenzel J, Uerlich M, Worrenkamper E, Freutel S, Bieber T, Tuting T. Scarring skin lesions of discoid lupus erythematosus are characterized by high numbers of skin-homing cytotoxic lymphocytes associated with strong expression of the type I interferon-induced protein MxA. Br J Dermatol 2005; 153: $1011-1015$

19 Bauer JW, Baechler EC, Petri M, Batliwalla FM, Crawford D, Ortmann WA, Espe KJ, Li W, Patel DD, Gregersen PK, Behrens TW. Elevated Serum Levels of Interferon-Regulated Chemokines Are Biomarkers for Active Human Systemic Lupus Erythematosus. PloS Med 2006; 3: e491

20 Kim JM, Rasmussen JP, Rudensky AY. Regulatory T cells prevent catastrophic autoimmunity throughout the lifespan of mice. Nat Immunol 2007; 8: 191 - 197

21 Franz B, Fritzsching B, Riehl A, Oberle N, Klemke $C D$, Sykora J, Quick S, Stumpf C, Hartmann M, Enk A, Ruzicka T, Krammer PH, Suri-Payer E, Kuhn A. Low number of regulatory T cells in skin lesions of patients with cutaneous lupus erythematosus. Arthritis Rheum 2007; 56: $1910-1920$

22 Crispin JC, Martinez A, Alcocer-Varela J. Quantification of regulatory T cells in patients with systemic lupus erythematosus. J Autoimmun 2003; 21: $273-276$

23 Banchereau J, Pascual V. Type I interferon in systemic lupus erythematosus and other autoimmune diseases. Immunity 2006; 25: 383-392

24 Casciola-Rosen L, Rosen A. Ultraviolet light-induced keratinocyte apoptosis: a potential mechanism for the induction of skin lesions and autoantibody production in LE. Lupus 1997; 6: 175-180

25 Kuhn A, Herrmann M, Kleber S, Beckmann-Welle M, Fehsel K, MartinVillalba A, Lehmann P, Ruzicka T, Krammer PH, Kolb-Bachofen V. Accumulation of apoptotic cells in the epidermis of patients with cutaneous lupus erythematosus after ultraviolet irradiation. Arthritis Rheum 2006; 54: 939-950

26 Baima B, Sticherling M. Apoptosis in different cutaneous manifestations of lupus erythematosus. Br J Dermatol 2001; 144: 958-966

27 Pablos JL, Santiago B, Galindo M, Carreira PE, Ballestin C, Gomez-Reino $J J$. Keratinocyte apoptosis and p53 expression in cutaneous lupus and dermatomyositis. J Pathol 1999; 188: 63-68

28 Reefman E, Kuiper H, Jonkman MF, Limburg PC, Kallenberg CG, Bijl M. Skin sensitivity to UVB irradiation in systemic lupus erythematosus is not related to the level of apoptosis induction in keratinocytes. Rheumatology (Oxford) 2006; 45: 538-544

29 Kaudewitz P, Ruzicka T, Meurer M, Rieber P, Braun-Falco O. Epidermal synthesis and expression of HLA-DR on keratinocytes in lupus erythematosus. Arch Dermatol Res 1985; 277: 444-447
30 Kuhn A, Sonntag M, Sunderkotter C, Lehmann P, Vestweber D, Ruzicka T. Upregulation of epidermal surface molecule expression in primary and ultraviolet-induced lesions of lupus erythematosus tumidus. $\mathrm{Br} \mathrm{J}$ Dermatol 2002; 146: 801 - 809

31 Miori L, Bellosta M, Rabbiosi G. HLA-DR positivity in discoid lupus erythematosus. J Am Acad Dermatol 1989; 21: 1316 - 1317

32 Tebbe B, Mazur L, Stadler R, Orfanos CE. Immunohistochemical analysis of chronic discoid and subacute cutaneous lupus erythematosus-relation to immunopathological mechanisms. Br J Dermatol 1995; 132: $25-31$

33 Meller S, Winterberg F, Gilliet M, Muller A, Lauceviciute I, Rieker J, Neumann NJ, Kubitza R, Gombert M, Bunemann E, Wiesner U, Franken-Kunkel P, Kanzler H, Dieu-Nosjean MC, Amara A, Ruzicka T, Lehmann P, Zlotnik $A$, Homey $B$. Ultraviolet radiation-induced injury, chemokines, and leukocyte recruitment: An amplification cycle triggering cutaneous lupus erythematosus. Arthritis Rheum 2005; 52: 1504- 1516

34 Furukawa F, Kashihara-Sawami M, Lyons MB, Norris DA. Binding of antibodies to the extractable nuclear antigens SS-A/Ro and SS-B/La is induced on the surface of human keratinocytes by ultraviolet light (UVL): implications for the pathogenesis of photosensitive cutaneous lupus. J Invest Dermatol 1990; 94: 77-85

35 Dorner T, Hucko M, Mayet WJ, Trefzer U, Burmester GR, Hiepe F. Enhanced membrane expression of the $52 \mathrm{kDa} \mathrm{Ro}(\mathrm{SS}-\mathrm{A})$ and $\mathrm{La}(\mathrm{SS}-\mathrm{B})$ antigens by human keratinocytes induced by TNF alpha. Ann Rheum Dis 1995; 54: 904-909

36 Gerl V, Hostmann B, Johnen C, Waka A, Gerl M, Schumann F, Klein R, Radbruch A, Hiepe F. The intracellular 52-kd Ro/SSA autoantigen in keratinocytes is up-regulated by tumor necrosis factor alpha via tumor necrosis factor receptor I. Arthritis Rheum 2005; 52: 531-538

37 Wittmann $M$, Werfel $T$. Interaction of keratinocytes with infiltrating lymphocytes in allergic eczematous skin diseases. Curr Opin Allergy Clin Immunol 2006; 6: 329-334

38 Wenzel J, Henze S, Worenkamper E, Basner-Tschakarjan E, SokolowskaWojdylo M, Steitz J, Bieber T, Tuting T. Role of the chemokine receptor CCR4 and its ligand thymus- and activation-regulated chemokine/ CCL17 for lymphocyte recruitment in cutaneous lupus erythematosus. J Invest Dermatol 2005; 124: 1241 - 1248

39 Limat A, French LE, Blal L, Saurat JH, Hunziker T, Salomon D. Organotypic cultures of autologous hair follicle keratinocytes for the treatment of recurrent leg ulcers. J Am Acad Dermatol 2003; 48: 207 - 214

40 Schwarz A, Stander S, Berneburg M, Bohm M, Kulms D, van Steeg H, Grosse-Heitmeyer K, Krutmann J, Schwarz T. Interleukin-12 suppresses ultraviolet radiation-induced apoptosis by inducing DNA repair. Nat Cell Biol 2002; 4: 26 - 31

41 Wozniacka A, Lesiak A, Narbutt J, McCauliffe DP, Sysa-Jedrzejowska A. Chloroquine treatment influences proinflammatory cytokine levels in systemic lupus erythematosus patients. Lupus 2006; 15: 268-275 